\title{
Single Stuck-At Fault Diagnosing Circuit of Reed-Muller Canonical Exclusive-Or Sum of Product Boolean Expressions
}

\author{
${ }^{1}$ P.N. Neelakantan and ${ }^{2}$ A. Ebenezer Jeyakumar \\ ${ }^{1}$ Electrical Engineering, Govt. College of Technology, Coimbatore-641 013, India \\ ${ }^{2}$ Govt. College of Engineering, Salem - 636 011, India
}

\begin{abstract}
A testable design with a universal test set for single stuck-at zero and stuck-at one faults of Reed-Muller canonical form of Exclusive-OR sum of product logic expressions is proposed. The test circuit detects almost all the single stuck-at faults and needs only simple modifications for variations in the circuit under test. The number of test vectors is also quite small compared with the classical method. The factor of un-identifiability is discussed and a new quantification parameter for the fault diagnosis has also been introduced. Results of Matlab simulations for a few logic functions are included.
\end{abstract}

Key words: Combinational circuits, exclusive-or sum of products, Reed-Muller canonical form, single stuck-at faults, testability realization, universal test set

\section{INTRODUCTION}

Any arbitrary binary logic function can be expressed as exclusive-or sum of product Reed-Muller canonical (ESOP RMC) form which results in minimal product terms as can be seen from Table $1^{[1]}$. The SOP is the conventional sum of product form while the other forms are variations of Reed-Muller canonical (RMC) expressions. The PPRM is the positive-polarity RMC form, which does not allow any complemented variable to occur in the expression. For example, $\mathrm{x} 1 \oplus \mathrm{x} 2 \mathrm{x} 3 \oplus$ $\mathrm{x} 4 \mathrm{x} 5 \mathrm{x} 6$ is a PPRM expression, while $\mathrm{x} 1{ }^{\prime} \oplus \mathrm{x} 2 \mathrm{x} 3$ is not. The FPRM allows negation of any variable, but throughout the expression the variable should appear only in the same form, either complemented or uncomplemented. Thus, $\mathrm{x} 11^{\prime} \oplus \mathrm{x} 2 \mathrm{x} 3 \oplus \mathrm{x} 1{ }^{\prime} \times 4$ is a FPRM expression since $\mathrm{x} 1$ is appearing as only complemented variable, whereas the expression $\mathrm{x} 1 \oplus$ $\mathrm{x} 2 \mathrm{x} 3 \oplus \mathrm{x} 1{ }^{\prime} \mathrm{x} 4$ is not FPRM as $\mathrm{x} 1$ is present in uncomplemented form in the first term and in complemented form in the third term. GRM is the abbreviation for Generalized Reed-Muller form. In this structure, a variable is free to appear as complemented or uncomplemented, but should should not result in same PPRM terms more than once. For instance, $\mathrm{x} 1 \oplus$ $\mathrm{x} 2 \mathrm{x} 3 \oplus \mathrm{x} 2{ }^{\prime}{ }^{\prime} 3^{\prime}$ is not a GRM since the term $\mathrm{x} 2{ }^{\prime} \times 3^{\prime}$ ' results in $\times 2 \times 3$ when converted into PPRM, which is already present as the second term. The Exclusive-Or Sum of Product (ESOP) form, on the other hand, does not impose any of the restrictions mentioned above and, in fact, is the most general form of RMC expressions. Such an expression is of the form $\mathrm{f}=\mathrm{a}_{0} \oplus \mathrm{a}_{1} \mathrm{x}_{1} * \oplus \mathrm{a}_{2} \mathrm{x}_{2} *$ $\ldots . . \oplus a_{n} x_{n} * \oplus a_{n+1} x_{1} * x_{2} * \oplus \ldots . . \oplus a_{2 n-1} x_{1} * x_{2} * \ldots x_{n} *$ where $x_{n} *$ can be $x_{n}$ or its negation and $a_{n}$ is either 0 or 1 . The main advantage of such a form, apart from minimal number of product terms, is that it enables a simple method of diagnosis ${ }^{[2-4]}$. They also provide a more efficient realization than conventional AND-OR functions in many applications such as linear circuits, arithmetic circuits and telecom networks ${ }^{[5]}$. Further, a more compact PLA implementation based on ANDEXOR form is achievable compared with the AND-OR circuits $^{[6]}$. The basic disadvantage of slow speed and greater chip area of exclusive-or based implementations has become less prominent, with the abundant availability of FPGA's since the last decade ${ }^{[7]}$. A ReedMuller canonical form of CMOS implementation can be easily tested for stuck-open faults with a universal test set ${ }^{[8]}$. Mixed polarity Reed-Muller expressions have also been useful in classification of Boolean functions ${ }^{[11]}$. In spite of the slow speed and larger chip area of RMC implementations compared to other others, some of the RMC forms require only a lesser area and also have been effectively used in the FPGA based modules of Xilinx, Actel ${ }^{[9]}$.

Table 1: Number of product terms of some arithmetic functions in different forms

\begin{tabular}{llllll}
\hline Function & SOP & PPRM & FPRM & GRM & ESOP \\
\hline adr4 & 75 & 34 & 34 & 34 & 31 \\
$\log 8$ & 123 & 253 & 193 & 105 & 96 \\
nrm4 & 120 & 216 & 185 & 96 & 69 \\
ndm8 & 76 & 56 & 56 & 31 & 31 \\
rot8 & 57 & 225 & 118 & 51 & 35 \\
sym9 & 84 & 210 & 173 & 126 & 51 \\
wgt8 & 255 & 107 & 107 & 107 & 58 \\
\hline
\end{tabular}

Corresponding Author: P.N. Neelakantan, Electrical Engineering, Govt. College of Technology, Coimbatore-641 013, India 
Literature survey: A classical method of generating test patterns for very large and complex logic functions is Linear Feedback Shift Register (LFSR) based pseudo-exhaustive or pseudo-random type ${ }^{[1]}$. However, this does not work well with ESOP form as shown by Drechsler et al. ${ }^{[10]}$.A PPRM network for detection of stuck-at faults with a universal test of size $n+4, n$ being the number of data inputs, was proposed by Reddy ${ }^{[2]}$. Though quite good for self-testing, the method is economical only for PPRM form, which obviously has more number of product terms than the other forms in most cases. Multiple stuck-at fault detection for ESOP circuits was carried out by Pradhan ${ }^{[11]}$. However since the cardinality is $2 \mathrm{n}+6+\sum \mathrm{nCe}, \mathrm{e}=0$ to $\mathrm{j}$, the order of ESOP expression, the test set is not universal and also is too large to be practical for large input functions. Stuck-at and bridging faults with a universal test set for PPRM has also been reported ${ }^{[12]}$. Multiple fault detecting GRM realizations was propounded by $\mathrm{Sasao}^{[4]}$. It was shown that $2 \mathrm{n}+\mathrm{s}+3$ test vectors, where $\mathrm{s}$ is the number of product terms in the logic function are required for single stuck-at fault detections in GRM circuit while $2 n+s$ vectors are required for detection of and/or bridging faults in GRM/ESOP circuits ${ }^{[13]}$. Here too, the test set is not universal as it depends on s, the number of product terms of the function. Kalay et al. ${ }^{[1]}$ described an ESOP implementation with a universal test set of size $n+6$ for single faults. A robust and universal sequence has been proposed for stuck-open type of faults in GRM/ESOP cmos transistor implementations ${ }^{[14]}$. Zhongliang ${ }^{[15]}$ demonstrated that the single stuck-at fault detection can be achieved with only $\mathrm{n}+5$ test vectors. Apart from a small modification in his circuit, two methods, each with minor modifications in his scheme, are proposed in this paper and results of matlab simulations for a few specific functions comparing the detectability of the faults have been included. Further, the concept of indistiguishability index has also been introduced and compared for the illustrative functions.

Network structure: The network structure of the proposed scheme is similar to that proposed by Zhongliang $^{[15]}$ and is shown in Fig.1. It comprises literal-complementing xor block, an AND block, an xor function tree block, which implements the required logic function as also two additional outputs o1 and 02 obtained through a separate AND and an OR gate. The actual data inputs to the system are $\mathrm{x}_{1}, \mathrm{x}_{2}, \ldots . \mathrm{x}_{\mathrm{n}}$. Additionally, the scheme requires four control inputs $\mathrm{c}_{0}$ to $c_{3}$. The literal-complementing block produces the complements of the literals used in the function. Only those literals appearing in complemented form require an xor gate in this block. The literals of each product term are combined through an AND gate and hence the number of AND gates required is the same as the number of product terms in the logic function. Further, each of the AND gates of this block may have an additional input from one of the control lines depending on the number of gates used in the xor tree block producing the final function $\mathrm{f}$. For a function requiring seven xor gates as shown in Fig. 2, the eight AND gates connected to the eight input lines of the xor tree will receive additional control lines respectively from $\mathrm{c}_{3}, \mathrm{c}_{1}$, $\mathrm{c}_{1}, \mathrm{c}_{2}, \mathrm{c}_{1}, \mathrm{c}_{2}, \mathrm{c}_{2}$ and $\mathrm{c}_{3}$ respectively. All the product terms are the passed on to the function xor tree block, which generates the required logic function f. Finally, all the data and control inputs are applied to a separate AND gate and an OR gate, producing auxiliary outputs o1 and o2, to aid in the detection of faults which cannot be differentiated by the main function output $f$ alone.

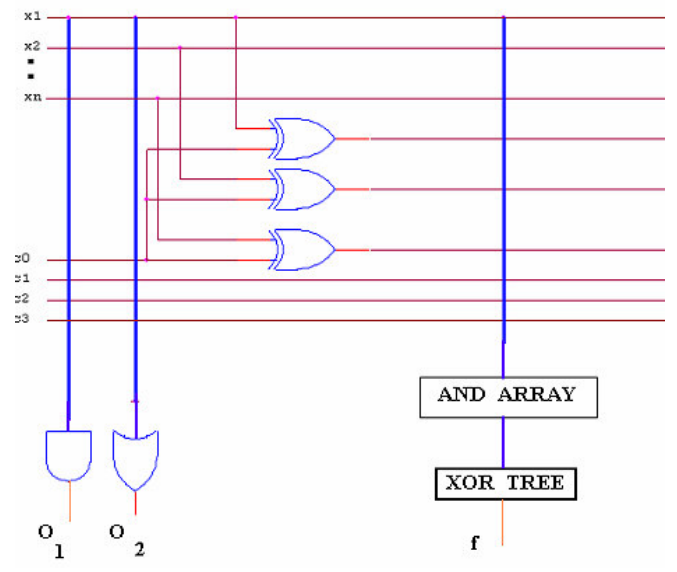

Fig. 1: Generalized network structure

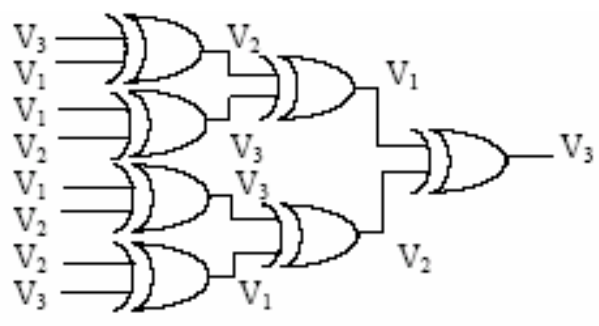

Fig. 2: An ex-or tree

Test vectors: Zhongliang ${ }^{[15]}$ proposed a test matrix for the detection of single stuck-at faults. Each of its rows is an $\mathrm{n}+4$ long vector, $\mathrm{n}$ being the number of data inputs. The first four columns of the matrix represent 
the control inputs $c_{0}$ to $c_{3}$ while the remaining $n$ columns that of the data inputs $\mathrm{x} 1$ to $\mathrm{xn}$. The first test

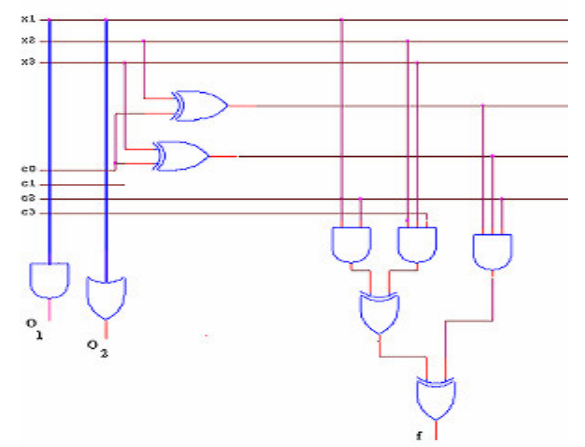

Fig. 3: Circuit for $\mathrm{f}=\mathrm{x}_{1} \oplus \mathrm{x}_{2} \mathrm{x}_{3} \oplus \mathrm{x}_{2} \mathrm{x}_{3}$,

vector is an all-zero vector. The second vector has only $\mathrm{c}_{0}$ and $\mathrm{c}_{1}$ as zero, while in the third vector only $\mathrm{c}_{0}$ and $\mathrm{c}_{2}$ are zero valued. The next vector consists of all 1 's except for $c_{0}$. The next $n$ vectors are made up of 'walking zero vectors'. Finally the last vector is an allzero one except for the first element. The test matrix is the same for any logic function in Reed-Muller ESOP form provided that the number of data inputs is the same.

\section{PROPOSED MODIFICATIONS AND SIMULATION RESULTS}

Instead of the second and third vectors with 'walking zero vectors' (or 'weezee vectors' to be short) fixed in columns $c_{1}$ and $c_{2}$, two modifications are now proposed: In one, which I prefer to call as 'AC weezee' method, all the control inputs participate in the 'zero walk'. The second method, which may be called as 'Alternative Vector Method' suggests $c_{2}$ and $c_{3}$ for 'weezee' instead of $c_{1}$ and $c_{2}$. The network structures and the test matrices for the reference method ${ }^{[15]}$ as well as the now proposed modifications were simulated in matlab. The single stuck-at faults of type s-a-0 and s-a1 , at each of the data and control inputs, the literalcomplementing xor gate outputs, the AND block outputs as also the function xor tree gate outputs were simulated by redefining the corresponding variable to zero or one. The test vectors were applied as the values of the simulated logic variables and the resulting outputs $\mathrm{f}, \mathrm{o} 1$ and $\mathrm{o} 2$, each as a vector of $n+5$ elements (for the reference method and Alternative Vector method) or $n+6$ elements (for AC weezee method) were then converted to the equivalent decimal values and tabulated for convenience and easy comparison. The simulation results are as follows:

Example1: $(\mathrm{n}=3) \mathrm{f}=\mathrm{x}_{1} \oplus \mathrm{x}_{2} \mathrm{x}_{3} \oplus \mathrm{x}_{2} \mathrm{x}_{3}$, $\mathrm{f}=$ Output of the function xor tree block

o1= Output of the separate AND gate

$\mathrm{o} 2=$ Output of the separate OR gate

\section{Reference method $^{[15]}$}

No fault $=>\{\mathrm{f}, \mathrm{o} 1, \mathrm{o} 2\}=\{118,112,127\}$

Single stuck-at fault at one of control inputs $c_{0}$ to $c_{3}$, or data inputs $\mathrm{x}_{1}$ to $\mathrm{x}_{3}$ :

Total number of possible faults: $7 \times 2=14$

Table 2: Decimal equivalents of the outputs for s-a-0 fault at each of input and control inputs for the reference method

\begin{tabular}{lcccrrrr}
\hline & $\mathrm{c}_{0}$ & $\mathrm{c}_{1}$ & $\mathrm{c}_{2}$ & $\mathrm{c}_{3}$ & $\mathrm{x}_{1}$ & \multicolumn{1}{c}{$\mathrm{x}_{2}$} & \multicolumn{1}{c}{$\mathrm{x}_{3}$} \\
\hline $\mathrm{f}$ & 118 & 118 & 120 & 14 & 32 & 86 & 86 \\
$\mathrm{o} 1$ & 112 & 112 & 112 & 112 & 0 & 0 & 0 \\
$\mathrm{o} 2$ & 126 & 127 & 127 & 127 & 127 & 127 & 127 \\
\hline
\end{tabular}

Table 3: Decimal equivalents of the outputs for s-a-1 fault at each of input and control inputs for the reference method

\begin{tabular}{lrcccrcc}
\hline & $\mathrm{c}_{0}$ & $\mathrm{c}_{1}$ & $\mathrm{c}_{2}$ & $\mathrm{c}_{3}$ & $\mathrm{x}_{1}$ & $\mathrm{x}_{2}$ & $\mathrm{x}_{3}$ \\
\hline $\mathrm{f}$ & 46 & 118 & 119 & 118 & 126 & 118 & 118 \\
$\mathrm{O} 1$ & 0 & 112 & 112 & 112 & 20 & 116 & 114 \\
$\mathrm{o} 2$ & 255 & 127 & 127 & 127 & 255 & 255 & 255 \\
\hline
\end{tabular}

Single stuck-at fault at one of intermediate gate outputs:

$\mathrm{zl}_{1}, \mathrm{zl}_{2}$ : Outputs of literal-complementing xor gates; $\mathrm{zl}_{1}$ equivalent to the complement $\mathrm{x}_{2}$, and $\mathrm{zl}_{2}$ for $\mathrm{x}_{3}$ ' given in the function.

$\mathrm{za}_{1}, \mathrm{za}_{2}, \mathrm{za}_{3}$ : Outputs of the AND block gates; $\mathrm{za}_{1}$ for the first product term $\mathrm{x}_{1} \mathrm{c}_{2}, \mathrm{za}_{2}$ for the second term $\mathrm{x}_{2} \mathrm{x}_{3} \mathrm{c}_{3}$ and $\mathrm{za}_{3}$ for the last term $\mathrm{x}_{2}, \mathrm{x}_{3}, \mathrm{c}_{2}$

$\mathrm{zX}_{1}, \mathrm{zx}_{2}$ : Outputs of the function xor tree gates; $\mathrm{zx}_{1}$ with $\mathrm{Za}_{1}$ and $\mathrm{za}_{2}$ as inputs, while $\mathrm{zX}_{2}(=\mathrm{f})$ with $\mathrm{Zx}_{1}$ and $\mathrm{Za}_{3}$ as inputs producing the final output.

Total number of possible faults: $(2+3+2) \times 2=14$

Table 4: Decimal equivalents of the outputs for s-a-0 fault at each of gate outputs for the reference method

\begin{tabular}{lrrrrrrr}
\hline & $\mathrm{zl}_{1}$ & $\mathrm{Zl}_{2}$ & $\mathrm{Za}_{1}$ & $\mathrm{Za}_{2}$ & $\mathrm{Za}_{3}$ & $\mathrm{ZX}_{1}$ & $\mathrm{Zx}_{2}(=\mathrm{f})$ \\
\hline $\mathrm{f}$ & 46 & 46 & 32 & 14 & 46 & 88 & 0 \\
$\mathrm{o} 1$ & 0 & 0 & 112 & 112 & 112 & 112 & 112 \\
$\mathrm{o} 2$ & 127 & 127 & 127 & 127 & 127 & 127 & 127 \\
\hline
\end{tabular}

Table 5: Decimal equivalents of the outputs for s-a-1 fault at each of gate outputs for the reference method

\begin{tabular}{llrrrrrc}
\hline & $\mathrm{zl}_{1}$ & $\mathrm{zl}_{2}$ & $\mathrm{za}_{1}$ & $\mathrm{za}_{2}$ & $\mathrm{za}_{3}$ & $\mathrm{zx}_{1}$ & $\mathrm{zX}_{2}(=\mathrm{f})$ \\
\hline $\mathrm{f}$ & 114 & 116 & 223 & 241 & 209 & 167 & 255 \\
$\mathrm{o} 1$ & 112 & 112 & 112 & 112 & 112 & 112 & 112 \\
$\mathrm{o} 2$ & 255 & 255 & 127 & 127 & 127 & 127 & 127 \\
\hline
\end{tabular}

Comments:

* Gross Total of possible single s-a-0 / s-a-1 faults: $14+14=28$.

* Identical outputs for 'No fault', sa0 / sa1 @ c1and 
sa1 @ c3 $\{\mathrm{f}=118, \mathrm{o} 1=112$ and $\mathrm{o} 2=127\}$

$\rightarrow \quad 3 / 28=10.71 \%$ completely unidentifiable

* Same outputs for the following faults:

$\mathrm{sa} 0 @ \mathrm{x}_{2} / \mathrm{x}_{3} \quad\{\mathrm{f}, \mathrm{o} 1, \mathrm{o} 2\}=\{86,0,127\}$

$\mathrm{sa} 0 @ \mathrm{zl}_{1} / \mathrm{zl}_{2} \quad\{\mathrm{f}, \mathrm{o} 1, \mathrm{o} 2\}=\{46,0,127\}$

$\mathrm{sa} 0 @ \mathrm{c} 3 / \mathrm{za}_{2} \quad\{\mathrm{f}, \mathrm{o} 1, \mathrm{o} 2\}=\{14,112,127\}$

$\rightarrow 6 / 28=21.43 \%$ indistinguishable

Proposed method: (Alternative vector method)

No fault $=>\{\mathrm{f}, \mathrm{o} 1, \mathrm{o} 2\}=\{86,0,127\}$

Single stuck-at fault at one of control inputs $c_{0}$ to $c_{3}$, or data inputs $\mathrm{x}_{1}$ to $\mathrm{x}_{3}$ : Total number of faults: $7 \mathrm{x} 2=14$

Table 6: Decimal equivalents of the outputs for s-a-0 fault at each of input and control inputs for the proposed method

\begin{tabular}{lrrrrrrr}
\hline & $\mathrm{c}_{0}$ & $\mathrm{c}_{1}$ & $\mathrm{c}_{2}$ & $\mathrm{c}_{3}$ & $\mathrm{x}_{1}$ & \multicolumn{1}{c}{$\mathrm{x}_{2}$} & \multicolumn{1}{c}{$\mathrm{x}_{3}$} \\
\hline $\mathrm{f}$ & 86 & 86 & 88 & 14 & 96 & 54 & 54 \\
$\mathrm{o} 1$ & 0 & 0 & 0 & 0 & 0 & 0 & 0 \\
$\mathrm{o} 2$ & 126 & 127 & 127 & 127 & 127 & 127 & 127 \\
\hline
\end{tabular}

Table 7: Decimal equivalents of the outputs for s-a-1 fault at each of input and control inputs for the proposed method

\begin{tabular}{lrrrrrrr}
\hline & $\mathrm{c}_{0}$ & $\mathrm{c}_{1}$ & $\mathrm{c}_{2}$ & $\mathrm{c}_{3}$ & $\mathrm{x}_{1}$ & $\mathrm{x}_{2}$ & \multicolumn{1}{c}{$\mathrm{x}_{3}$} \\
\hline $\mathrm{f}$ & 110 & 86 & 87 & 118 & 94 & 86 & 86 \\
$\mathrm{o} 1$ & 16 & 0 & 0 & 0 & 0 & 0 & 0 \\
$\mathrm{o} 2$ & 255 & 255 & 255 & 255 & 255 & 255 & 255 \\
\hline
\end{tabular}

Single stuck-at fault at one of intermediate gate outputs: Total number of possible faults: $(2+3+2) \times 2=14$

Table 8: Decimal equivalents of the outputs for s-a-0 fault at each of gate outputs for the proposed method

\begin{tabular}{lrrrrrrr}
\hline & $\mathrm{zl}_{1}$ & $\mathrm{zl}_{2}$ & $\mathrm{za}_{1}$ & $\mathrm{za}_{2}$ & $\mathrm{za}_{3}$ & $\mathrm{zx}_{1}$ & $\mathrm{zx}_{2}=\mathrm{f}$ \\
\hline $\mathrm{f}$ & 110 & 110 & 96 & 14 & 110 & 56 & 0 \\
$\mathrm{o} 1$ & 0 & 0 & 0 & 0 & 0 & 0 & 0 \\
$\mathrm{o} 2$ & 127 & 127 & 127 & 127 & 127 & 127 & 127 \\
\hline
\end{tabular}

Table 9: Decimal equivalents of the outputs for s-a-1 fault at each of gate outputs for the proposed method

\begin{tabular}{lrrrrrrr}
\hline & $\mathrm{Zl}_{1}$ & $\mathrm{Zl}_{2}$ & $\mathrm{Za}_{1}$ & $\mathrm{Za}_{2}$ & $\mathrm{Za}_{3}$ & $\mathrm{ZX}_{1}$ & $\mathrm{ZX}_{2}(=\mathrm{f})$ \\
\hline $\mathrm{f}$ & 82 & 84 & 159 & 241 & 145 & 199 & 255 \\
$\mathrm{o} 1$ & 0 & 0 & 0 & 0 & 0 & 0 & 0 \\
$\mathrm{o} 2$ & 127 & 127 & 127 & 127 & 127 & 127 & 127 \\
\hline
\end{tabular}

\section{Comments:}

* Gross total of possible single s-a-0 / s-a-1 faults: $14+14=28$.

* Identical outputs for 'no fault', sa0 @ c1 $\{\mathrm{f}, \mathrm{o} 1, \mathrm{o} 2\}=\{86,0,127\}$

$\rightarrow 1 / 28=3.57 \%$ completely unidentifiable fault

* Same outputs for

$\begin{array}{ll}\mathrm{sa} 0 @ \mathrm{c} 4 / \mathrm{za} 2 & (14,0,127) \\ \mathrm{sa} 0 @ \mathrm{x} 1 / \mathrm{za} 1 & (96,0,127) \\ \mathrm{sa} 0 @ \mathrm{x} 2 / \mathrm{x} 3 & (54,0,127) \\ \mathrm{sa} 1 @ \mathrm{c} 2 / \mathrm{x} 2 / \mathrm{x} 3 & (86,0,255) \\ \mathrm{sa} 0 @ \mathrm{z} 11 / \mathrm{z} 12 / \mathrm{za} 3 & (110,0,127) \\ 28=42.86 \% \text { indistinguishable }\end{array}$

Table 10: Comparison of simulation results for a few logic functions

\begin{tabular}{|c|c|c|c|c|c|c|c|c|c|c|}
\hline \multirow{3}{*}{$\begin{array}{l}\text { Example } \\
\text { No. }\end{array}$} & \multirow{3}{*}{$\begin{array}{l}\text { No. of Data } \\
\text { inputs }\end{array}$} & \multirow{3}{*}{$\begin{array}{l}\text { Total } \\
\text { faults }\end{array}$} & \multirow{2}{*}{\multicolumn{2}{|c|}{$\begin{array}{l}\text { Reference } \\
\text { Method }^{[15]}\end{array}$}} & \multicolumn{6}{|c|}{ Proposed Methods } \\
\hline & & & & & \multicolumn{2}{|c|}{$\begin{array}{l}\text { Reference } \\
\text { Method Vector }\end{array}$} & \multicolumn{2}{|c|}{$\begin{array}{l}\text { AC weezee } \\
\text { Vector }\end{array}$} & \multicolumn{2}{|c|}{$\begin{array}{l}\text { Alternative } \\
\text { Vector }\end{array}$} \\
\hline & & & $\% \mathrm{U}$ & $\% \mathrm{I}$ & $\% \mathrm{U}$ & $\% \mathrm{I}$ & $\% \mathrm{U}$ & $\% \mathrm{I}$ & $\% \mathrm{U}$ & $\% \mathrm{I}$ \\
\hline 1 & 3 & 28 & 10.71 & 21.43 & 3.57 & 46.43 & 3.57 & 42.86 & 3.57 & 42.86 \\
\hline 2 & 3 & 28 & 10.71 & 21.43 & 3.57 & 50 & 3.57 & 46.43 & 3.57 & 46.43 \\
\hline 3 & 3 & 30 & 7.14 & 20 & 7.14 & 50 & 7.14 & 42.86 & 7.14 & 42.86 \\
\hline 4 & 4 & 32 & 9.38 & 21.88 & 3.13 & 43.75 & 3.13 & 40.63 & 3.13 & 40.63 \\
\hline 5 & 5 & 38 & 2.63 & 23.68 & NIL & 55.26 & NIL & 44.74 & NIL & 44.74 \\
\hline 6 & 6 & 38 & 10.53 & 28.95 & 2.63 & 52.63 & 2.63 & 50 & 2.63 & 50 \\
\hline 7 & 7 & 40 & 7.5 & 35 & 2.5 & 47.5 & 2.5 & 42.5 & 2.5 & 45 \\
\hline 8 & 8 & 54 & NIL & 35.19 & NIL & 44.44 & NIL & 44.44 & NIL & 44.44 \\
\hline 9 & 9 & 52 & 1.92 & 34.62 & NIL & 46.15 & Nil & 46.15 & NIL & 48.08 \\
\hline
\end{tabular}

U -- Unidentifiable I -- Indistinguishable

Example No. 1: $\mathrm{f}=\mathrm{x} 1 \oplus \mathrm{x} 2 \mathrm{x} 3 \oplus \mathrm{x} 2{ }^{\prime} \mathrm{x} 3$ '

Example No. 2: $\mathrm{f}=\mathrm{x} 1 \oplus \mathrm{x} 1 \mathrm{x} 2 \mathrm{x} 3 \oplus \mathrm{x} 2^{\prime} \times{ }^{\prime}{ }^{\prime}$

Example No. 3: $\mathrm{f}=\mathrm{x} 1^{\prime} \oplus \mathrm{x} 1^{\prime} \times 2^{\prime} \oplus \mathrm{x} 2 \mathrm{x} 3{ }^{\prime}$

Example No. 4: $\mathrm{f}=\mathrm{x} 1 \mathrm{x} 2 \mathrm{x} 3 \oplus \mathrm{x} 2 \mathrm{x} 3 \mathrm{x} 4 \oplus \mathrm{x} 2^{\prime} \mathrm{x} 3^{\prime} \times 4^{\prime}$

Example No. 5: $\mathrm{f}=\mathrm{x} 1 \mathrm{x} 5 \oplus \mathrm{x} 1 \mathrm{x} 2 \mathrm{x} 3 \oplus \mathrm{x} 2 \mathrm{x} 3 \mathrm{x} 44^{\oplus} \mathrm{x} 2{ }^{\prime} \mathrm{x} 3{ }^{\prime} \mathrm{x} 4^{\prime}$

Example No. 6: $\mathrm{f}=\mathrm{x} 1 \mathrm{x} 2 \mathrm{x} 6{ }^{\prime} \oplus \mathrm{x} 2 \mathrm{x} 3 \mathrm{x} 4 \oplus \mathrm{x}^{\prime}{ }^{\prime} \times 4^{\prime}{ }^{\prime} 5^{\prime}{ }^{\prime}$

Example No. 7: $\mathrm{f}=\mathrm{x} 1 \mathrm{x} 2 \mathrm{x} 7{ }^{\prime} \oplus \mathrm{x} 3 \mathrm{x} 4 \mathrm{x} 5 \mathrm{x}^{\prime} \mathrm{x}^{\prime} \times{ }^{\prime} 5^{\prime} \times 6^{\prime}$

Example No. 8: $\mathrm{f}=\mathrm{x} 1 \mathrm{x} 2 \mathrm{x} 8^{\prime} \oplus \mathrm{x} 3 \mathrm{x} 7^{\prime} \times 6^{\prime} \oplus \mathrm{x} 4^{\prime} \times 5^{\prime} \oplus \mathrm{x} 1^{\prime} \times 2^{\prime} \times 3^{\prime}$

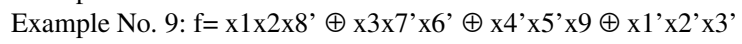


A similar procedure is adopted for the reference method vector ${ }^{[15]}$ as well as 'AC weezee' method, with proposed modified circuit. The results for the above as also a few additional examples are shown in Table 10.

\section{CONCLUSION}

Three test set schemes for detection of single stuck-at faults for logic functions have been proposed and the simulation results show that the proposed schemes reduce the possibility of unidentifiable faults. Further an additional index, the indistiguishability of faults, which is different from unidentifiability has also been proposed and compared for the example functions.

\section{REFERENCES}

1. Kalay, U., D.V. Hall and M.A. Petrowski, 2000. A minimal universal test set for self-test of EXORSum-of-Products circuits. IEEE Trans. Computers, 49: 267-276.

2. Reddy, S.M., 1972. Easily testable realizations for logical functions. IEEE Trans. Computers, 21: 1183-1188.

3. Saluja, K.K. and S.M. Reddy, 1975. Fault detecting test set for Reed-Muller canonic networks. IEEE Trans. Computers, 24: 995-998.

4. Sasao, T., 1997. Easily testable realizations for Reed-Muller expressions. IEEE Trans. Computers, 21: 709-716.

5. Aborhey, S., 2001. Reed-Muller tree-based minimization of fixed polarity Reed-Muller expansions. IEE Proc. Comput. Digit. Tech., 148: 2.

6. Sasao, T. and P. Besslich, 1990. On the complexity of mod-2 sum PLAs. IEEE Trans. Computers, 39: 262-266.
7. Wu, H. et al., 1996. Generalized partially-mixedpolarity Reed-Muller expansion and its fast computation. IEEE Trans. Computer, 45: 10841088.

8. Das, D.K., S. Chakraborty and B.B. Bhattacharya, 2003. Universal and robust testability of stuckopen faults in Reed-Muller canonical cmos circuits. Intl. J. Electron., 90: 1-11.

9. Wu, H. et al., 1996. Generalized partially-mixedpolarity Reed-Muller expansion and its fast computation. IEEE Trans. Computer, 45: 10841088.

10. Drechshler, R. et al., 1997. Testability of 2 level AND/EXOR Circuits. Proc. European Design and Test Conf.

11. Pradhan, D.K., 1978. Universal test sets for multiple fault detection in AND-EXOR arrays. IEEE Trans. Computers, 27: 181-187.

12. Bhattacharya, B.B. et al., 1985. Testable design of RMC networks with universal tests for detecting stuck-at and bridging faults. IEE Proc., 132 Part E: 155-161.

13. Zhongliang, P., 2003. Bridging fault detections for testable realizations of logic functions. Proc. of Intl. Conf. on VLSI Design, pp: 423-427.

14. Rahaman, H., D.K. Das and B.B. Bhattacharya, 2004. Testing of stuck-open faults in generalised Reed Muller and EXOR sum of products cmos circuits. IEEE Proc. Comput. and Digit. Tech., 151: 1.

15. Zhongliang, P., 2002. Testable realizations of ESOP expressions of logic functions. Proc. of 11th Asian Test Symposium (ATS”02), IEEE Computer Society. 